\title{
Raoultella ornithinolytica Diagnosed in a Neurointensive Patient. A Rare Case with Recovery without Antibiotics
}

\author{
Marlene Ersgaard Jellinge* \\ Department of Anesthesiology and Intensive Care Medicine, Odense University Hospital, Denmark
}

\begin{abstract}
Infection with Raoultella ornithinolytica is rare and normally the infection is present in patients with underlying malignancies or chronic diseases. It is normally treated with antibiotics. In this case report, a neuro-intensive patient without malignancies or other severe chronic diseases was colonized with Pseudomonas aeruginosa but infected with Raoultella ornithinolyca. The patient recovered without treatment with antibiotics.
\end{abstract}

Keywords: adult intensive care, Raoultella ornithinolytica, neuroanesthesia

Received: 5 January 2017 / Accepted: 5 May 2017

\section{INTRODUCTION}

Infection with Raoultella ornithinolytica is rare and this Gram-negative, oxidase-negative, non-motile, capsulated facultative anaerobic bacillus of the enterobacteriaceae family is normally found in aquatic environments, fish and insects [1-11].

Raoultella ornithinolytica occurs mainly in patients with underlying malignancies or chronic diseases, trauma, nosocomial infection and invasive procedures and is a risk factor for a poor outcome $[1,4,8-9,12]$. The bacterium was originally named Klebsiella ornithinolytica but was subsequently reclassified as Raoultella ornithinolytica. It is an important histamine-producing bacterium associated with distinctive cutaneous signs consisting of flushing or a maculopapular rash $[3,6-$ 7,10-13].

Raoultella ornithinolytica is a rarely reported hospital-acquired infection [9]. It is occasionally isolated from bronchial lavage or other deep respiratory samples and should never be considered simply a saprophytic bacterium. It shows a high resistance to antimicrobial resistance [9-10] and it has been reported that it may cause a fatal neonatal infection, especially in preterm infants [12].

The present report concerns a patient in a neurointensive care unit who had been diagnosed with the colonization of the trachea by Raoultella ornithinolytica and who recovered without antibiotic treatment. Written informed consent to publish this report was received from the patient and her husband.

\section{CASE REPORT}

A 48-year-old woman with hypertension was admitted to the emergency department in Odense University Hospital, Odense, Denmark, with subarachnoid haemorrhage associated with the internal carotid artery. She was treated with a stent and coiling technique. After a period of rehabilitation in hospital, she made significant progress and was discharged.

Two months later she suffered a second subarachnoid hemorrhage with bleeding into the brain ventricles and hydrocephalus due to a dissection of an aneurysm in the internal carotid artery. She was treated using the endovascular coiling technique. She was admitted to the Department of Neuro-intensive Care in the same hospital and sedated with propofol $9 \mathrm{ml} / \mathrm{h}(20 \mathrm{mg} / \mathrm{ml})$. Later this treatment was changed and she was sedated with sodium thiopental $7 \mathrm{ml} / \mathrm{h}(25 \mathrm{mg} / \mathrm{ml})$, fentanyl 6 $\mathrm{ml} / \mathrm{h}(50 \mathrm{mcg} / \mathrm{ml})$ and midazolam $10 \mathrm{ml} / \mathrm{h}(50 \mathrm{mg} / \mathrm{ml})$. An external ventricular drain was placed together with a Camino ${ }^{\circ}$ Micro Ventricular Bolt Intracranial Pressure Temperature Monitoring Kit (Integra Camino, San Diego, USA). 
Endovascular coiling was undertaken and twentyfive days after readmission she underwent a percutaneous dilatational tracheostomy to provide a long-term route for mechanical ventilation. The sedation procedure was reduced over time and she slowly made progress. Maintaining normal blood pressure was a challenge but otherwise she had no other organ dysfunctions.

Four days after the tracheostomy, a positive test was obtained for multi-resistant Pseudomonas from samples from her trachea. A decision was taken to change the tracheostomy tube and to isolate the patient as part of the infection control regime. Furthermore, it was elected only to prescribe antibiotics if symptoms of infection occurred. The tracheostomy tube was changed to a Bivona ${ }^{\circledR}$ Adult TTSTM tracheotomy size 7.0 with cuff (Smiths Medical, Kent, UK).

On day thirty-seven after readmission, and nine days after the change of the tracheostomy tube, the patient became febrile. There was no evidence of flushing or rash. A blood transfusion had not been given and the laboratory data revealed a C-reactive protein level of 15 $\mathrm{mg} / \mathrm{L}$ and a white blood cell count of $17.5 \times 10^{9} / \mathrm{L}$ (Table 1, Laboratory data). However, Gram-negative bacilli were cultured from the patient's trachea, along with Pseudomonas. The hospital's clinical department of microbiology identified these as Raoultella ornithinolytica using the matrix-assisted laser desorption/ionization time-of-flight mass spectrometry (MALDI-TOF MS) (Bruker Daltronics Biotyper 3.1). Microbe identification and antibiotic susceptibility tests were performed by disk diffusion and the organism was shown to be sensitive to cefuroxime, gentamicin and ciprofloxacin but resistant to ampicillin. The next day a new sample was sent to the microbiology department and Raoultella ornithinolytica was identified for the second time, still together with a high number of Pseudomonas. This time they were resistant to ampicillin and piperacillin/ tazobactam. The recommendation was to start treatment with antibiotics only if she showed symptoms of infection. In accordance with this advice, antibiotics were withheld and on day three and four after the first test result with Raoultella ornithinolytica, tracheal swabs were found to be negative.

Forty-nine days after the second subarachnoid hemorrhage and eleven days after the identification of Raoultella ornithinolytica, the patient was able to breathe spontaneously. The tracheostomy tube was removed and she was discharged from the intensive care unit to a general ward. The patient made slow but continuous progress. She was paralyzed on the left side of her body, suffered from nausea and headaches, found it difficult to concentrate and spoke only a few words. Later she was discharged to a neurological rehabilitation clinic where she had regained almost all of her neurocognitive functions although she still had memory problems. At the end of the rehabilitation program she was discharged to her home.

\section{DIsCUSSION}

Most case reports of Raoultella ornithinolytica concern patients with serious comorbidities. In one study, the mortality rate related to infection was found to be $5 \%$ [10].

The patient in the present case had a previous history of arterial hypertension but no malignancies or other chronic diseases and was otherwise in good physical condition, fit and non-smoking. This differs from the descriptions of patients reported to be infected with Raoultella ornithinolytica [3,5,7-11-13], though in a case report concerning a 21-day-old boy, the patient was healthy except for having a sinus venous type atrial septal defect [12].

In other case reports patients received antibiotics, unlike the present case where the patient was not treated with antibiotics but nevertheless recovered [3,5,713]. Details of this watch-and-wait policy have not been found in the literature.

In a previous study [10], Raoultella ornithinolytica pneumonia was observed in twenty patients over a ninety-one-month period. Half of these cases had hospital-acquired pneumonia and three had ventilator-

Table 1. Laboratory data from the department of clinical biochemistry and clinical microbiology

\begin{tabular}{lcccccc} 
Date & $\mathbf{1 4 / 4}$ & $\mathbf{1 5 / 4}$ & $\mathbf{1 6 / 4}$ & $\mathbf{1 7 / 4}$ & $\mathbf{1 8 / 4}$ & $\mathbf{1 9 / 4}$ \\
C-reactive Protein mg/L & 9.5 & 13 & 15 & 28 & 14 & 18 \\
White blood cell x 109/L & 9.0 & 14.0 & 17.5 & 9.0 & 8.7 & 12.6 \\
Temperature ${ }^{\circ} \mathrm{C}$ & 36.9 & 37.2 & 37.5 & 37.8 & 37.4 & 37.0 \\
Raoultella ornithinolytica & Negative & Negative & Positive & Positive & Negative & Negative \\
\hline
\end{tabular}


associated pneumonia. The patients diagnosed with Raoultella ornithinolytica presented a medical history of solid cancer, immunodeficiency, diabetes mellitus or chronic alcoholics [10]. This seems to be different from the woman in the present case report.

Identification of Raoultella species is difficult due to its phenotypic similarity to Klebsiella species [1,911]. The MALDI-TOF MS technique, used to identify the bacterium in this case, has a high sensitivity and specificity for Raoultella ornithinolytica. The technique has been used to correctly and rapid identify of various microorganisms, including Raoultella ornithinolytica which often do not need confirmation by molecular procedures [9-10].

Accurate identification of Raoultella ornithinolytica and determination of antibiotics susceptibility are necessary to determine appropriate patient care. Normally Raoultella ornithinolytica strains are sensitive to at least two or three different antibiotics [5], but one report has described that a strain was resistant to all antibiotics except tigecycline and colistin [14]. Raoultella species have the potential for multi-drug resistance [4]. The consensus recommendation is that a Raoultella ornithinolytica infection should be treated with antibiotics e.g. amoxicillin plus clavulanic acid for 10-14 days [6].

\section{CONCLUSION}

This is a case report concerning a patient in the neurointensive care who was infected with Raoultella ornithinolytica and the distinctive feature in this case was that the patient was successfully treated without antibiotics, suggestive of the advantage offered by a "watch-andwait policy".

Raoultella ornithinolytica is a pathogen with potent virulence. It is very rare but should be dealt with attention. Raoultella ornithinolytica may have fatal outcome in patients with severe comorbidities.

The prevalence of Raoultella ornithinolytica may be an underreported, emerging hospital-acquired infection and early recognition and proper infection control measures are important. Physicians should be aware of the possibility of medical and surgical device-associated Raoultella ornithinolytica infections and the risk of antimicrobial resistance.

\section{CONFLICT OF INTEREST}

None declared.

\section{REFERENCES}

1. Chun S, Yun JW, Huh HJ, Lee NY. Clinical characteristics of Raoultella ornithinolytica bacteriemia. Infection. 2015;43(1):59-64.

2. Haruki Y, Hagiya H, Sakuma A, et al. Clinical characteristics of Raoultella ornithinolytica bacteremia: a case series and literature review. J Infect Chemother. 2014;20(9):589-91.

3. Mau N, Ross LA. Raoultella ornithinolytica bacteremia in an infant with visceral heterotaxy. Pediatr Infect Dis J. 2010;29(5):477-8.

4. Boattini $M$, Almeida $A$, Cardoso $C$ et al. Infections on the rise: Raoultella spp., clinical and microbiological findings from a retrospective study, 2010-2014. Infect Dis (Lond). 2016;48(1):87-91.

5. Kabbara WK, Zgheib YR. Diabetic foot infection caused by Raoultella ornithinolytica. Am J Health Syst Pharm. 2015;72(24):2147-9.

6. Morais VP, Daporta MT, Bao AF, Campello MG, Andrés $\mathrm{GQ}$. enteric fever-like syndrome caused by Raoultella ornithinolytica (Klebsiella ornithinolytica). J Clin Microbiol. 2009;47:868-9.

7. Hadano Y, Tsukahara M, Ito K, et al. Raoultella ornithinolytica bacteremia in cancer patients: report of three cases. Intern Med. 2012;51(22):3193-5.

8. Kaya S, Bayramoğlu G, Sönmez M, Köksal í. Raoultella ornithinolytica causing fatal sepsis. Braz J Infect Dis. 2015;19(2):230-1.

9. Sękowska A, Dylewska K, Gospodarek E, Bogiel T. Catheterrelated blood stream infection caused by Raoultella ornithinolytica. Folia Microbiol (Praha). 2015;60(6):493-5.

10. Seng $P$, Boushab BM, Romain $F$ et al. Emerging role of Raoultella ornithinolytica in human infections: a series of cases and review of the literature. Int J Infect Dis. 2016;45:6571.

11. Yamakawa K, Yamagishi Y, Miyata K et al. Bacteremia caused by Raoultella ornithinolytica in two children. Pediatr Infect Dis J. 2016;35(4):452-3.

12. Ozkan EA, Khosroshahi HE, Yilmaz N, Gecit UA, Domur E. A rare case of a newborn with urinary tract infection by Raoultella ornithinolytica. American Journal of Health Research. 2014;2(6):375-7.

13. Solak Y, Gul EE, Atalay H, Genc N, Tonbul HZ. A rare human infection of Raoultella ornithinolytica in a diabetic foot lesion. Ann Saudi Med. 2011;31(1):93-4

14. Khajuria A, Praharaj AK, Grover N, Kumar M. First report of blaNDM-1 in Raoultella Ornithinolytica. Antimicrob Agents Chemother. 2013;57:1092-3. 